\title{
INHALT
}

Yzcatqui in innenonotzaliztlatol in ixquichtin teteo $\ldots \ldots \ldots \ldots \ldots \ldots \ldots \ldots \ldots \ldots$

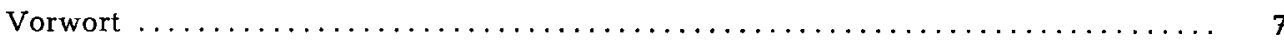

Gedanken über den ethnologischen Wert der Indianerforschung $\ldots \ldots \ldots \ldots \ldots \ldots \ldots$

José T u d ela, Madrid

Notas de historia económica. Los gallos de dos mundos $\ldots \ldots \ldots \ldots \ldots \ldots \ldots \ldots \ldots \ldots$

Heinrich U b b lo h de - D o e ring, Gossfelden bei Marburg/Lahn

Eine Parallele zwischen Alt-Peru und Alt-Mexiko $\ldots \ldots \ldots \ldots \ldots \ldots \ldots \ldots \ldots \ldots$

\section{MEXIKO UND MITTELAMERIKA}

Frans B 1 o m, San Cristóbal Las Casas, Chiapas/México

Historical Notes Relating to the Pre-columbian Amber Trade from Chiapas ........ 24

Mary B u t l e r, Philadelphia

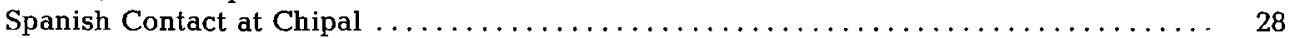

Rebeca Carrión Ca ch ot de Girard, Guatemala

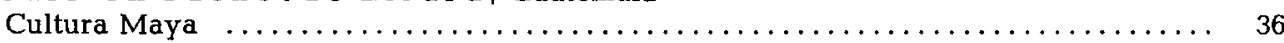

Alfonso C a s o, México, D.F.

El Dios 1. Muerte ........................................ 40

Carmen C ook de L e o n a rd, México, D.F.

Archäologisch-geographische Probleme der Insel Jaina, Campeche, Mexiko . . . . . . . 44

Guda E. G. v a n G i f f e n - D u y vis, Groningen Einige Musikinstrumente aus dem Staat Colima, Mexiko ................ 48

Wolfgang $\mathrm{Ha}$ be r l and, Hamburg

Zentral-Amerika: Begriff, Grenzen und Probleme $\ldots \ldots \ldots \ldots \ldots \ldots \ldots \ldots \ldots \ldots$

Josef $\mathrm{H}$ a e k e l, Wien

Der ${ }_{\text {"Herr der Tiere }}$ im Glauben der Indianer Mesoamerikas . . . . . . . . . . . . . 60

Alfred V. Kidd e r and Edwin M. Shook, Cambridge, Mass./U.S.A.

A Unique Ancient Maya Sweathouse, Guatemala $\ldots \ldots \ldots \ldots \ldots \ldots \ldots \ldots \ldots \ldots \ldots$

Paul K i r c h h of f, México, D.F.

Las dos rutas de los colhua entre Tula y Culhuacán $\ldots \ldots \ldots \ldots \ldots \ldots \ldots \ldots \ldots \ldots$

Henri L e h m a $\mathbf{n}$, Paris

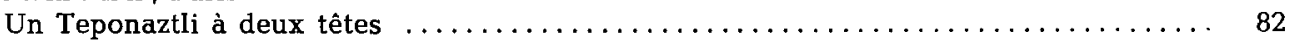

Samuel K. L o th r op, Cambridge. Mass./U.S.A.

A Re-appraisal of Isthmian Archaeology $\ldots \ldots \ldots \ldots \ldots \ldots \ldots \ldots \ldots \ldots \ldots \ldots \ldots$

J. Alden Ma s o n, Philadelphia The Tepehuan of Northern Mexico $\ldots \ldots \ldots \ldots \ldots \ldots \ldots \ldots \ldots \ldots \ldots \ldots \ldots \ldots \ldots \ldots$

Karl Anton Now otn y, Wien Die Hieroglyphen des Codex Mendoza. Der Bau einer mittelamerikanischen Wortschrift

Adrián R e c in o s, Guatemala

Algunas ideas sobre el origen de las razas indígenas de Guatemala . . . . . . . . 114

Doris Stone, San José de Costa Rica

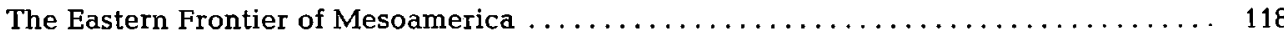

J. Eric S. Th o m p s o n, Ashdon Saffron Walden, Essex/England

The Role of Caves in Maya Culture . 
Hermann Trim b o r $\mathbf{n}$, Bonn

Rechtsgebräuche der Cueva in Panama $\ldots \ldots \ldots \ldots \ldots \ldots \ldots \ldots$

Hasso v o n W i n $\mathbf{n}$ in g, Hollywood, Calif./U.S.A.

Eine keramische Dorfgruppe aus dem alten Nayarit im westlichen Mexiko . . . . . . . 138

Otto Z er r ies, München

Wildgeister und Jagdritual in Zentralamerika

III. SUD - UND NORDAMERIKA

Herbert B a l d u s, São Paulo

Beiträge in deutscher Sprache zur Indianerforschung in Brasilien (1954-1958) .

Thomas S. B a r the l, Tübingen

Neuere Arbeiten zur Ethnographie und Archäologie Chiles $\ldots \ldots \ldots \ldots \ldots$

Hans B e c h e r, Hamburg

Xelekuhahé. Das Stockduell der Surára- und Pakidái-Indianer. Ein Beitrag zum

Problem der "Nilotenstellung" und der Tonsur in Südamerika

Hans D i t s c h y, Basel

Das Häuptlingswesen bei den Karaja' . . . . . . . . . . . . . . . . . . . 168

Karin $\mathrm{H}$ is s in k, Frankfurt/Main

Die Medizinmann-Trommel der Tacana $\ldots \ldots \ldots \ldots \ldots \ldots$

Ivar Pa 1 s on, Rotebro/Schweden

Zur Aufbewahrung der Tierknochen im nördlichen Nordamerika

182

Literaturverzeichnis $\quad \ldots \ldots \ldots \ldots \ldots \ldots \ldots \ldots \ldots \ldots \ldots \ldots \ldots \ldots \ldots \ldots$

Ubertragung des aztekischen Textes von $\mathrm{S} .6 \ldots \ldots \ldots \ldots \ldots$ 